\title{
Simulation of the edge plasma in tokamaks
}

\author{
Coster, D.P. ${ }^{\mathrm{a}}$, Bonnin, X. ${ }^{\mathrm{b}}$, Braams, B ${ }^{\mathrm{c}}$ Reiter, D. ${ }^{\mathrm{d}}$, \\ Schneider, R. ${ }^{\mathrm{b}}$, and the ASDEX Upgrade Team \\ ${ }^{a}$ Max-Planck-Institut für Plasmaphysik, EURATOM Association, Garching, \\ Germany \\ b Max-Planck-Institut für Plasmaphysik, EURATOM Association, Greifswald, \\ Germany \\ ${ }^{\mathrm{c}}$ Courant Institute, New York University, New York, NY, USA \\ ${ }^{\mathrm{d}}$ Institut für Plasmaphysik, FZ Jülich, EURATOM Association, Jülich, Germany
}

\begin{abstract}
Edge plasma modelling is discussed with particular emphasis on the comparison of a fluid neutral model with a kinetic neutral model. By iterative inclusion of additional effects, the agreement between the two is improved. Of particular importance is the proper treatment of neutrals at the core boundary, of the proper implementation of a neutral flux limit, and the modification of the ion heat boundary condition to include the neutral contribution. In the end, the agreement in the upstream profiles of electron and ion temperatures, and of electron and core neutral densities is very satisfactory. In addition, the effects of parallel ion and electron heat flux limiters, of transport ballooning to the low field side, and of drifts are investigated.
\end{abstract}

\section{Introduction}

Transport simulations of the edge plasma are inherently (at least) two dimensional calculations that need to include effects arising from the plasma, from the neutrals that arise when the plasma recombines at a surface or in the volume, and the interaction of both the plasma and the neutrals with material surfaces.

Two treatments of the neutrals are in use in edge codes: (1) a fluid treatment, or (2) a kinetic treatment. The kinetic treatment tends to be more exact while the fluid treatment tends to be faster. The usual kinetic treatment of the neutrals by a Monte-Carlo approach also introduces the issue of Monte-Carlo noise and raises questions about proving "convergence" of the final results. Convergence with a fluid treatment is easier to demonstrate. 
What this paper addresses is the differences for one particular ASDEX Upgrade shot between the two approaches, and examines parameters within the neutral model that can be used to improve the agreement between the two models. We start by comparing a coupled B2-Eirene (i.e. fluid plasma/MonteCarlo neutrals) simulation with a limited set of experimental data to show that we are in the right "ball-park". We then do a matching B2 simulation with the fluid neutral model. In order to examine the effects of the various inputs to the fluid neutral model, most of the runs are done with a pure deuterium solution, and that on a coarser mesh than used for the B2-Eirene simulation. Finally the "new" settings are used to redo the fluid neutral B2 simulations on the finer mesh with impurities.

\section{Initial B2-Eirene run compared to experiment}

We start with a B2-Eirene simulation $(\mathrm{D}+\mathrm{C}+\mathrm{He})$ of an L-mode AUG shot. (B2-Eirene [1-4] is a code that couples together the fluid plasma code, B2 [510] and the Monte-Carlo neutrals code, Eirene [11].) The upstream profiles are matched quite well, figure 1, and the downstream profiles reasonably, figures 2 and 3.
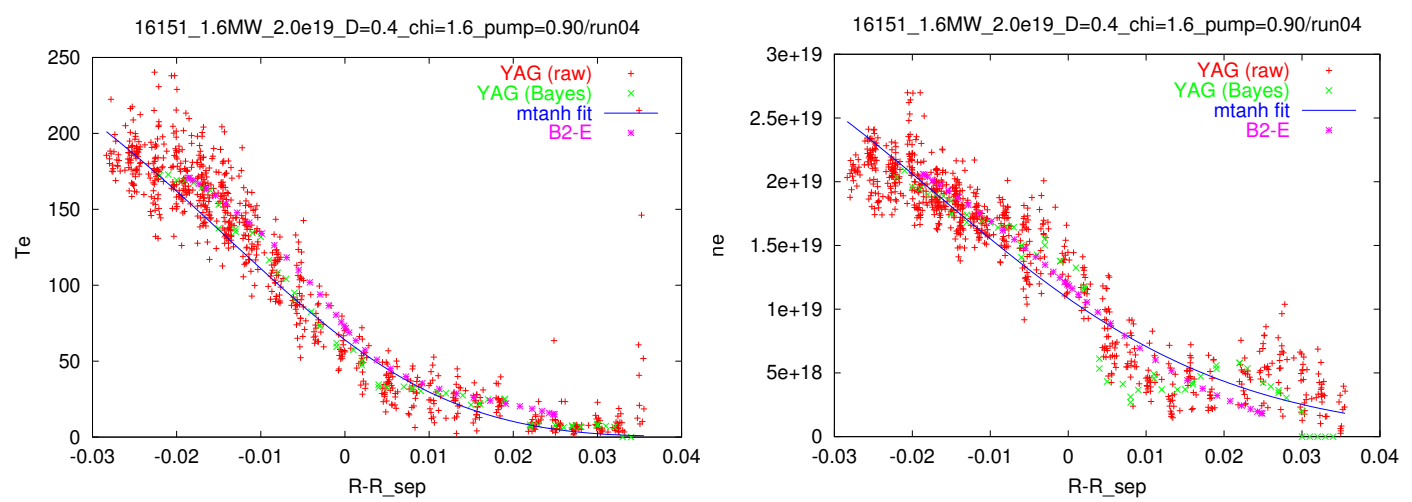

Fig. 1. Upstream electron temperature (left) and density (right) profiles. The raw YAG data, Bayesian filtered YAG data, the "mtanh" fit and the output from the B2-Eirene code are shown.

Normal sheath boundary conditions are used at the targets. The core boundary had 0.8 MW into each of the electrons and ions and a $D^{+}$density of $2 \times$ $10^{19} \mathrm{~m}^{-3} \cdot D^{+}$ions recycle at the SOL and private flux boundaries determined by a loss factor proportional to the product of the local sound speed and ion density. Carbon arises self-consistently from physical and chemical sputtering, has zero recycling at the targets and private flux and SOL boundaries, and has zero flux through the core boundary. A trace amount of helium is also present. $1 \mathrm{~cm}$ decay lengths were used for the decay lengths of the electron 

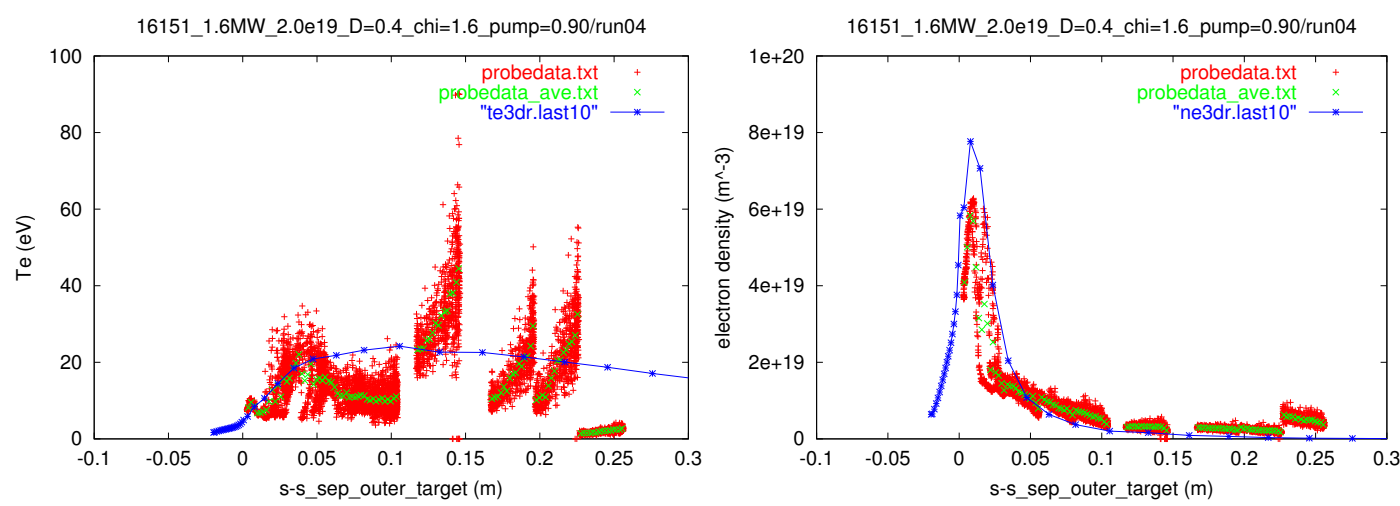

Fig. 2. Downstream electron temperature (left) and density (right) profiles. The raw data from the Langmuir probes, an experimental "average" and the B2-Eirene code results are shown.
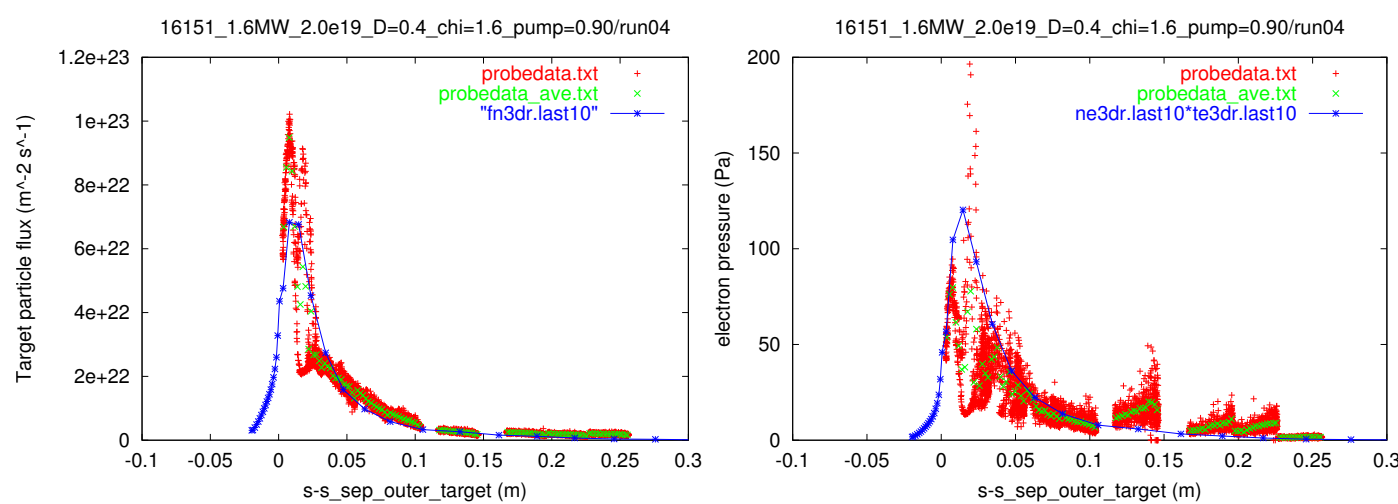

Fig. 3. Downstream saturation current (left) and electron pressure (right) profiles. The raw data from the Langmuir probes, an experimental "average" and the B2-Eirene code results are shown.

and ion temperatures at the SOL and private flux boundaries. No drifts were switched on for most of the runs.

The transport coefficients were $0.4 \mathrm{~m}^{2} \mathrm{~s}^{-1}$ for the ion particle diffusivities, and $1.6 \mathrm{~m}^{2} \mathrm{~s}^{-1}$ for the electron and ion thermal diffusivities.

\section{$3 \quad$ B2 with fluid neutrals}

We then try a B2 run without Eirene, using a fluid model for the neutrals. This shows systematic differences in the upstream profiles, figure 4 . In order to understand the differences, D only runs were performed on the same $96 \times 36$ mesh and on a $48 \times 18$ mesh. While the electron temperatures are similar, the ion temperature for the B2 cases is systematically lower than for the B2-Eirene case, the B2 electron densities are slightly larger, and the neutral densities are 
significantly larger. The B2 cases show very little difference arising from the grid size, and, in these quantities, only a small difference between the $\mathrm{D}+\mathrm{C}+\mathrm{He}$ and D-only cases.
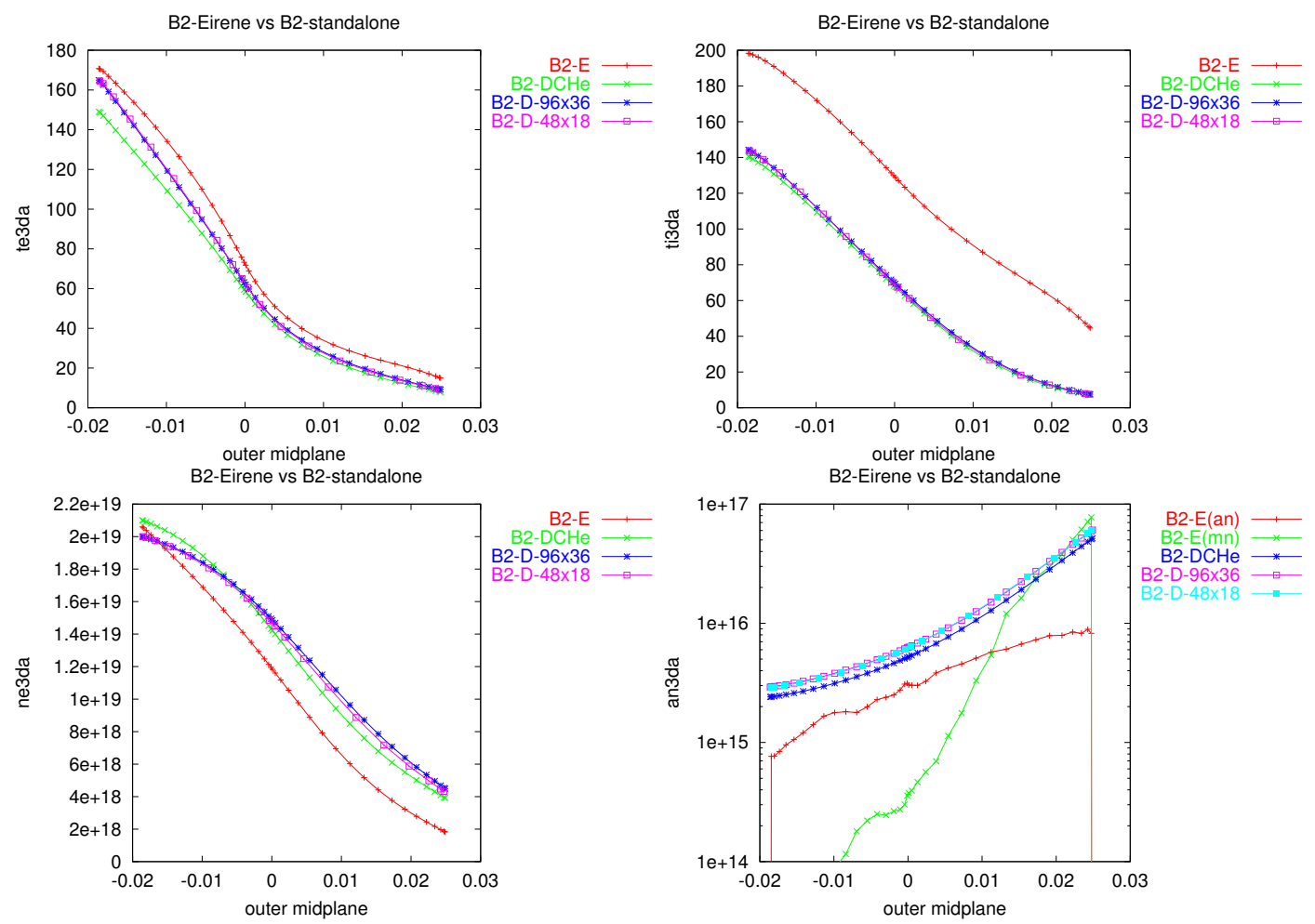

Fig. 4. Upstream electron temperature, ion temperature, electron density and neutral density. Data from a B2-Eirene run is compared to three B2 runs, with $\mathrm{D}+\mathrm{C}+\mathrm{He}$ on the same mesh, and then D on the same (96x36) mesh and a coarser mesh (48x18). Shown are the electron temperature ('te3da") in $e V$, the ion temperature ("ti3da") in $e V$, the electron density ("ne3da") in $m^{-3}$ and the neutral density ("an3da") in $m^{-3}$, all at the outer midplane with zero corresponding to the separatrix (and the distance given in $\mathrm{m}$ ). For the B2-Eirene calculation, two contributions to the neutral density are given, the atomic ("an") and molecular ("mn") contributions the fluid neutral model only has a model for the atom contribution.

The reason for the investigation of the $\mathrm{D}$ versus $\mathrm{D}+\mathrm{C}+\mathrm{He}$ and $96 \times 36$ versus $48 \times 18$ differences was simply that it is much faster to run D-48x18 than $\mathrm{D}+\mathrm{C}+\mathrm{He}-96 \times 36$ cases. The subsequent investigation of the B2-Eirene versus B2 difference is done with D-only on the reduced grid.

In figure 5 we compare the four "standard" cases (B2-Eirene-D+C+He-96x36, B2-D +C+He-96x36, B2-D-96x36 and B2-D-48x18) with three cases, all B2D-48x18 with "2e-2" an increased pumping of neutrals at the private flux boundary, "cnl" a change of the core neutral density boundary condition from zero flux to a loss flux proportional to the product of the local sound speed and the local neutral density, and "kiD0-X0.1" a neutral thermal diffusivity 

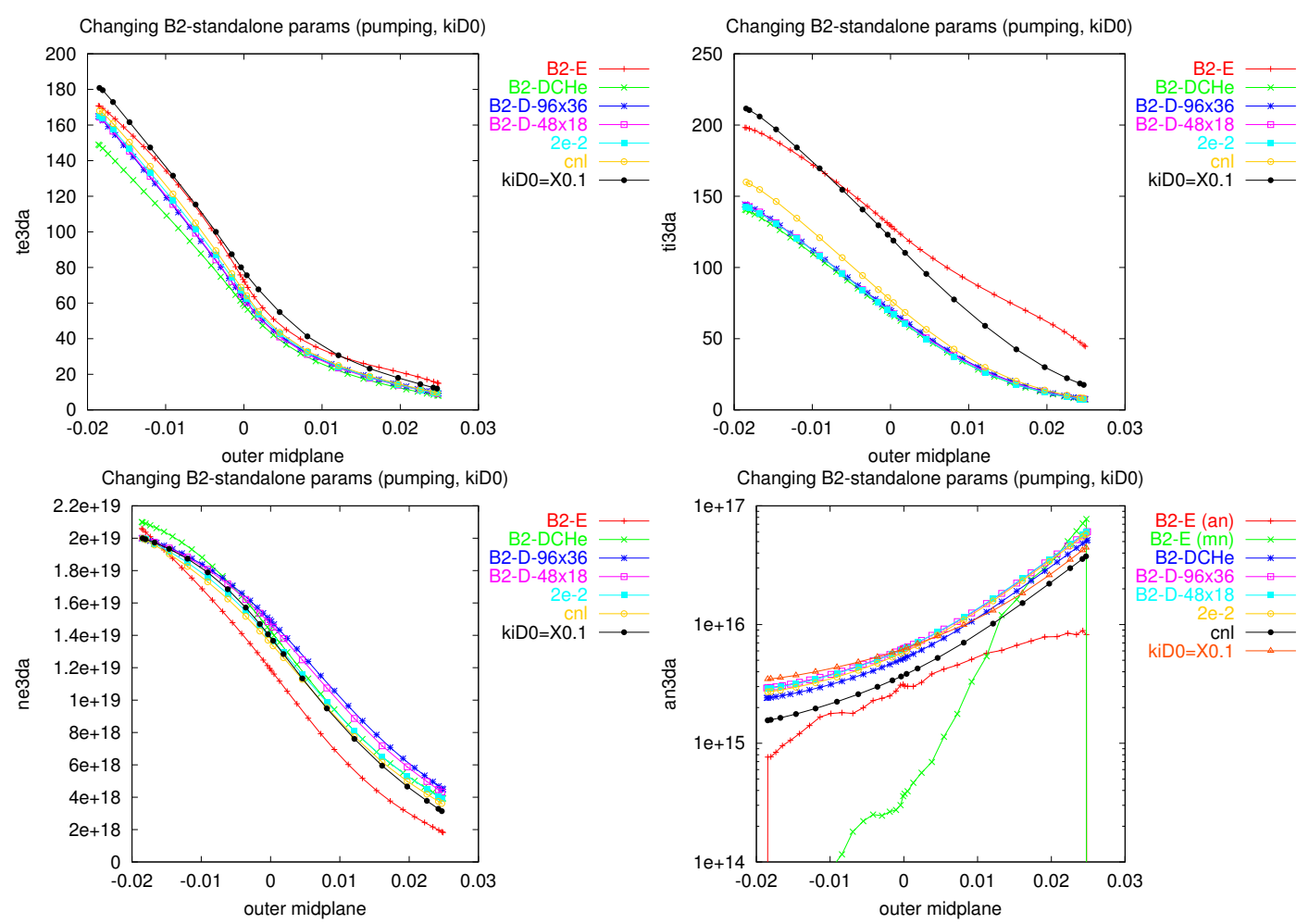

Fig. 5. Upstream electron temperature, ion temperature, electron density and neutral density. Data from the B2-Eirene run and the three B2 runs (with $\mathrm{D}+\mathrm{C}+\mathrm{He}$ on the same mesh, and then D on the same $(96 \times 36)$ mesh and a coarser mesh $(48 \times 18))$, compared with three B2-D-48x18 runs, doubled the pumping ( $2 \mathrm{e}-2$ "), core neutral loss model ("cnl") and the neutral thermal diffusivity ("kiD0-X0.1") .

reduced by a factor of 10 . In comparing the ion temperature profiles, we see the the "kiD0-X0.1" variation has the largest effect, the "cnl" variation a smaller effect and the "2e-2" variation a negligible effect. In the other profiles, we see a large effect in the neutral density for the "cnl" case.

The next set of variations arises from the introduction of a neutral flux limit [12], which has two parts: "fnb" limiting the convective flux of neutrals to some fraction of the local sound speed of the neutrals, and "lh0" limiting the conducted heat flux carried by the neutrals to some fraction of the sound speed of the neutrals times the local neutral energy content. In figure 6 we compare our standard set of plots with six cases where the neutral flux limit model was varied. In four cases, both "fnb" and "lh0" were varied together, for values of $0.3,1.0,2.0$ and 5.0. Two further cases had only one of the terms active, with the value 1.0. We see that of the two pieces to the neutral flux limiter, that piece contributing to the neutral thermal diffusivity seems to be the more important. Comparing the four different cases where both pieces were set, the best match overall seems to be for the "fln $=1.0$ " case. However, what is seen is a sharp drop of the ion temperature close to the outer boundary, and an 

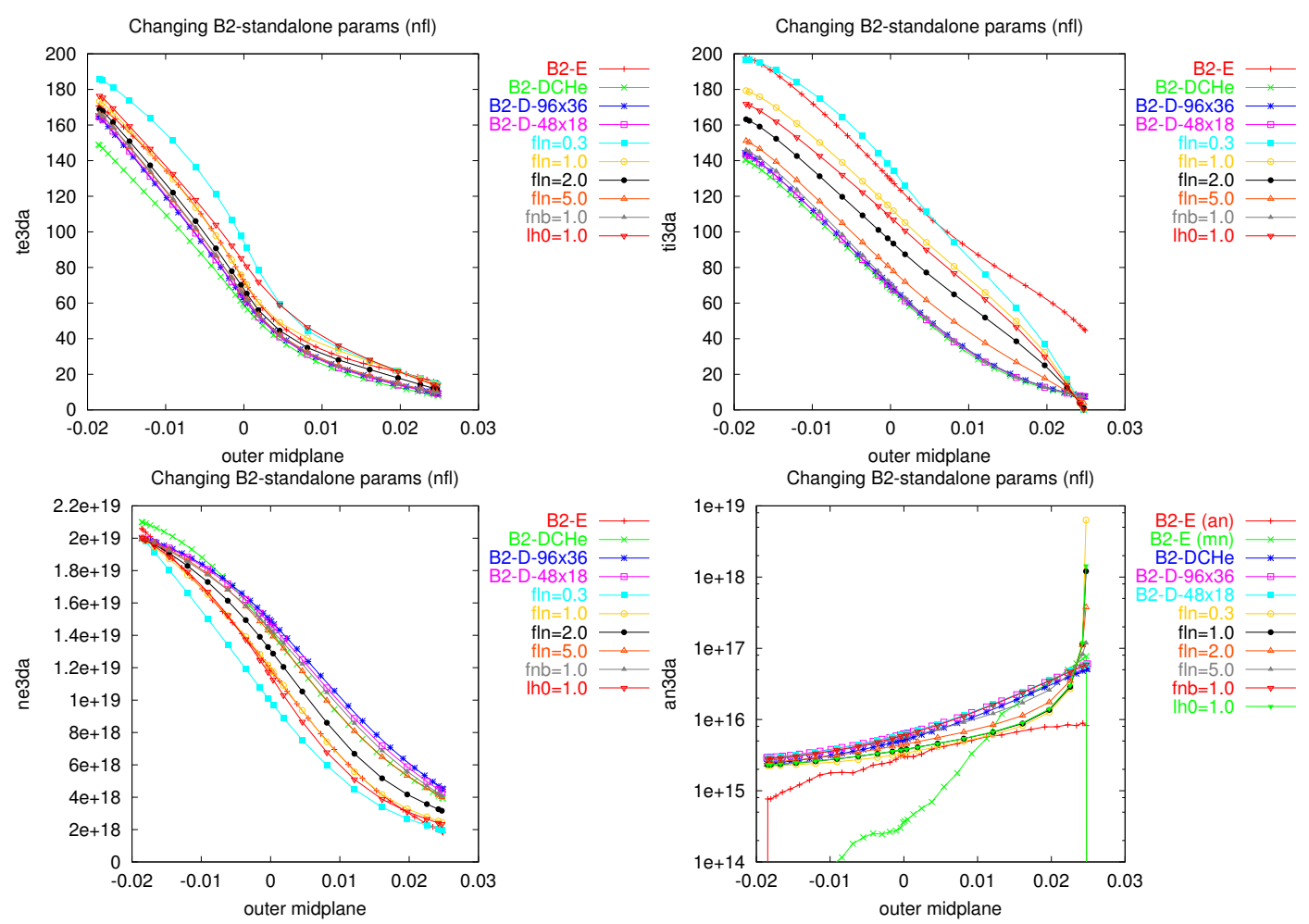

Fig. 6. Upstream electron temperature, ion temperature, electron density and neutral density. Data from the B2-Eirene run and the three B2 runs (with $\mathrm{D}+\mathrm{C}+\mathrm{He}$ on the same mesh, and then D on the same (96x36) mesh and a coarser mesh $(48 \times 18))$ are compared with six B2-D-48x18 runs, varying the neutral flux limit model. Four cases, " $\mathrm{fln}=0.3$, " $\mathrm{fln}=1.0 ", " \mathrm{fln}=2.0 "$ and " $\mathrm{fln}=5.0 "$ correspond to changing both pieces of the neutral flux limiters together, and two, "fnb=1.0" and "lh0=1.0" correspond to setting only one of the two pieces of the neutral flux limit.

associated sharp rise in the neutral density in that region.

In order to try and eliminate this anomalously low ion temperature at the outer boundary, additional runs were performed, figure 7 . Since this new feature was introduced by the neutral flux limiter model, the model was enhanced by the introduction of a minimum ion temperature which is used when calculating the neutral flux limiter. Runs were performed with this limit set to 10, 20 and $40 \mathrm{eV}$. While not eliminating the new ion temperature feature, the enhancement of the model did raise the edge ion temperature somewhat but overall a disappointing result. As another attempt to eliminate the anomalously low edge ion temperature, the first flight model in B2 was enabled this, instead of starting neutrals at the boundary, handles a first "kinetic" flight and passes to B2 a volume source of neutrals after the first charge exchange reaction. However this attempt also failed in significantly increasing the edge ion temperature.

Rather than changing the neutral flux limiter model, a different approach was 

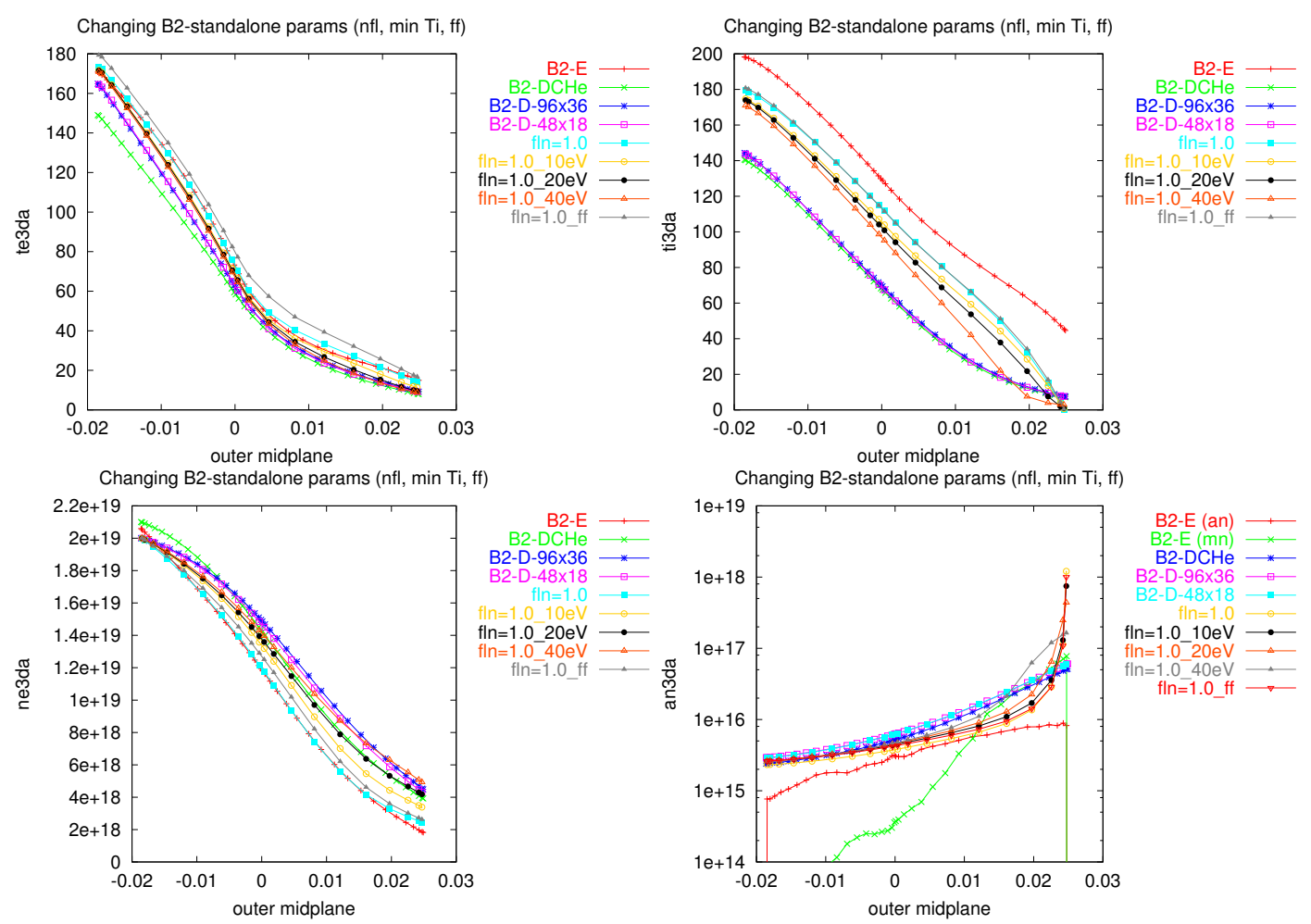

Fig. 7. Upstream electron temperature, ion temperature, electron density and neutral density. Data from the B2-Eirene run and the three B2 runs (with $\mathrm{D}+\mathrm{C}+\mathrm{He}$ on the same mesh, and then D on the same (96x36) mesh and a coarser mesh $(48 \times 18))$ are compared with five B2-D-48x18 runs, varying some features of the neutral model. The "fln=1.0" case from the previous figure is shown together with three variants where the neutral flux limiter model is augmented by imposing a minimum ion temperature in the limiting expression, " $\mathrm{fln}=1.0 \_10 \mathrm{eV}$ ", " $\mathrm{fln}=1.0 \_20 \mathrm{eV}$ " and "fln=1.0_40eV" (with respectively minimum temperatures in the flux limiter of 10, 20 and $40 \mathrm{eV}$ ), and a case where the first flight model in B2 is enabled.

tried next - that of modifying the boundary conditions associated with the outer SOL from those that had been used for the B2-Eirene calculation (and for the previous B2 runs). For the electron and ion temperatures a decay length of $1 \mathrm{~cm}$ had been specified, and for the $D^{+}$ions, a loss proportional to the product of the local sound speed of the ions and the local ion density (which was then recycled as neutrals). The variants shown in figure 8 change these in turn: "fln $=\mathbf{1 . 0} \mathbf{l} \mathbf{l}=\mathbf{0 . 1}$ " changed the electron temperature decay length to $10 \mathrm{~cm}$, "fln=1.0_li=0.1" changed the ion temperature decay length to $10 \mathrm{~cm}$, and "fln=1.0_1e-4" reduced the proportionality constant for the ion loss by a factor of 10 . We immediately see that the "fln $=1.0 \_\mathrm{li}=0.1$ " variant achieves what we want - a dramatic increase in the edge ion temperature.

The reason for this change can also be understood: in the coupled B2-Eirene case the "ion" thermal transport is carried only by the ions; in the standalone B2 runs with the fluid neutrals, "ion" energy is also transported by the 

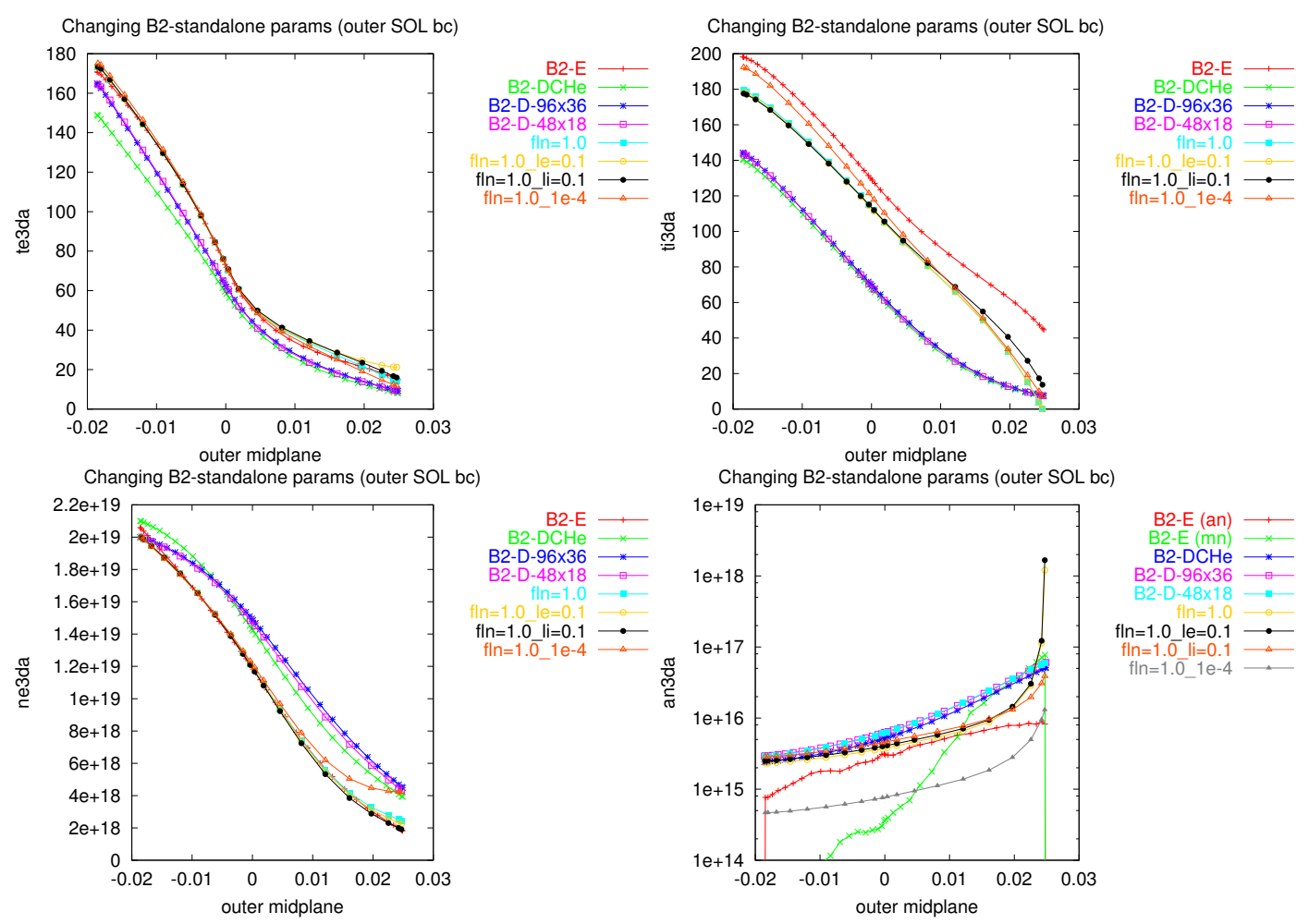

Fig. 8. Upstream electron temperature, ion temperature, electron density and neutral density. Data from the B2-Eirene run and the three B2 runs (with $\mathrm{D}+\mathrm{C}+\mathrm{He}$ on the same mesh, and then D on the same $(96 \times 36)$ mesh and a coarser mesh $(48 \times 18))$ are compared with four B2-D-48x18 runs with the neutral flux limiter model, but varying the SOL edge boundary conditions. The " $\mathrm{fn}=1.0$ " case is the same as presented previously. The " $\mathrm{fln}=1.0 \_\mathrm{le}=0.1$ " and " $\mathrm{fln}=1.0 \_\mathrm{li}=0.1$ " are variants where the temperature decay lengths for the electrons and ions, respectively, were changed from the $1 \mathrm{~cm}$ that had been used for the B2-Eirene and previous runs to $10 \mathrm{~cm}$. In the final variant, "fln=1.0_1e-4", the loss rate of $D^{+}$ions (giving rise to recycling) was made 10 times smaller.

neutrals (which, in the model, have the same temperature as the ions), and in the edge region is, without the neutral flux limiter, the dominant contributor. The neutral flux limiter limits the neutral contribution, but the neutrals are still the dominant player at the edge. The sharp feature we were seeing at the edge was a mismatch between the bulk transport and the edge condition imposed by the boundary condition - relaxing the imposed gradient length produced a better match between the bulk and the boundary.

In the next set of runs, shown in figure 9 , the match for four different choices of the ion temperature decay length are shown: "fln=1.0" with $1 \mathrm{~cm}$ (the value used in the B2-Eirene run with kinetic neutrals), " $\mathbf{f l n}=\mathbf{1 . 0} \mathbf{l} \mathbf{i}=\mathbf{0 . 1}$ " with $10 \mathrm{~cm}$, 'fln=1.0_li=0.2'" with $20 \mathrm{~cm}$, and 'fln=1.0_li=0.5" with $50 \mathrm{~cm}$. The best match seems to be somewhere between 20 and $50 \mathrm{~cm}$. 

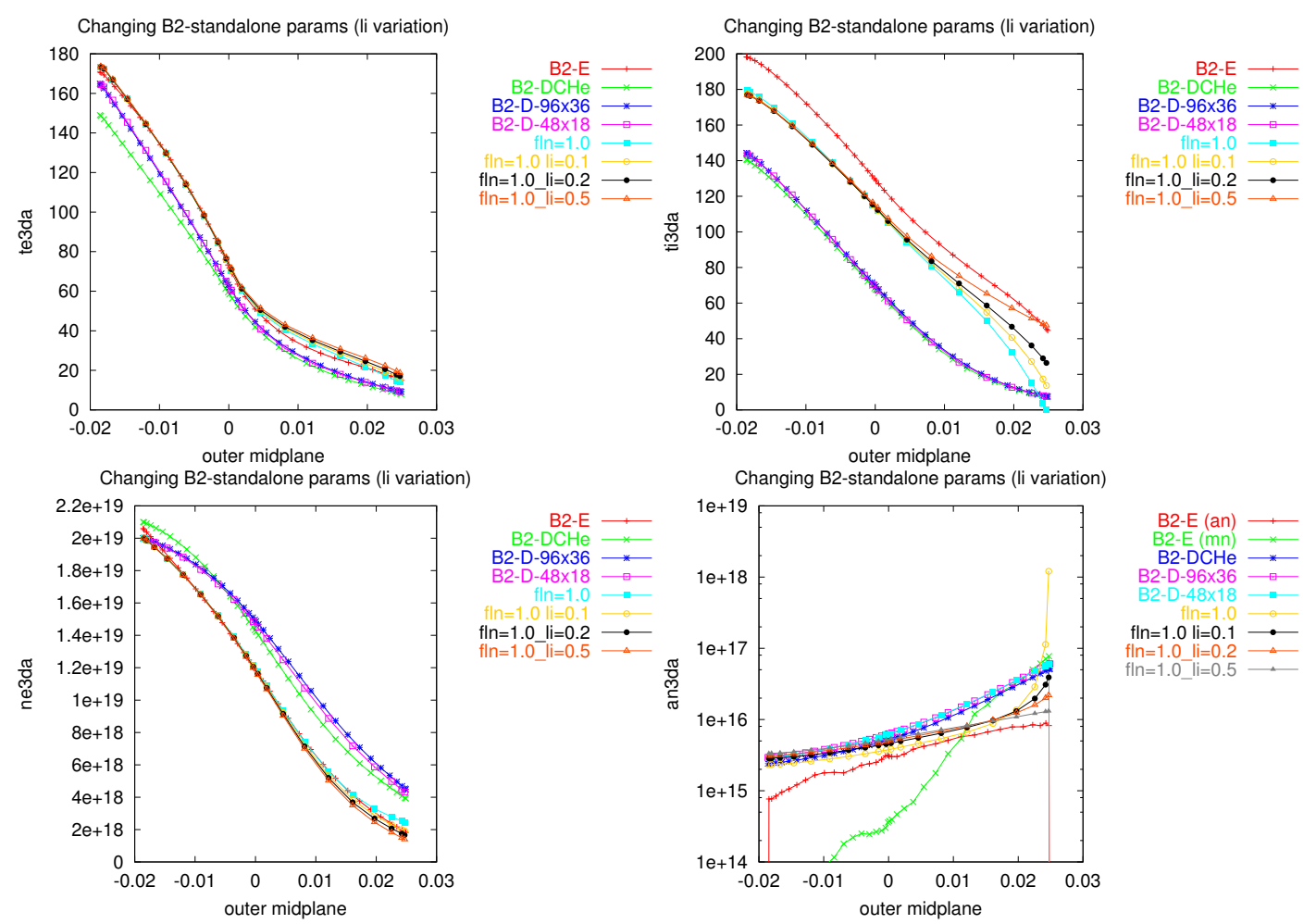

Fig. 9. Upstream electron temperature, ion temperature, electron density and neutral density. Data from the B2-Eirene run and the three B2 runs (with $\mathrm{D}+\mathrm{C}+\mathrm{He}$ on the same mesh, and then D on the same $(96 \times 36)$ mesh and a coarser mesh $(48 \times 18))$ are compared with four B2-D-48x18 runs all with the same neutral flux limit model, but varying ion temperature decay length. The "fln=1.0" case (shown in multiple previous graphs) has a decay length of $1 \mathrm{~cm}$; " $\mathrm{fln}=1.0 \_\mathrm{li}=0.1 ", " \mathrm{fln}=1.0 \_\mathrm{li}=0.2$ " and "fln=1.0_li=0.5" have decay lengths, respectively, of 10, 20 and $50 \mathrm{~cm}$.

The various, previously explored options, can be combined, and a selection are shown in figure 10. The combinations are: "fln=1.0" with a neutral flux limiter with value 1.0 (as reference); "fln=1.0 $\mathbf{l i}=\mathbf{0 . 1}$ " with a neutral flux limiter with value 1.0 with ion temperature decay length of $10 \mathrm{~cm}$; “... cnl" with a neutral flux limiter with value 1.0 with ion temperature decay length of $10 \mathrm{~cm}$ and neutrals lost to the core; "... cnl kiD0=X0.5" with a neutral flux limiter with value 1.0 with ion temperature decay length of $10 \mathrm{~cm}$, neutrals lost to the core and the neutral thermal diffusivity reduced by a factor of 2 ; “... cnl ff" with a neutral flux limiter with value 1.0 with ion temperature decay length of $10 \mathrm{~cm}$, neutrals lost to the core and the first flight model; “... cnl $10 \mathbf{e V}^{\prime}$ " with a neutral flux limiter with value 1.0 with ion temperature decay length of $10 \mathrm{~cm}$, neutrals lost to the core and the minimum temperature in the neutral thermal flux limiter set to $10 \mathrm{eV}$; and "... cnl $10 \mathrm{eV}$ ff" with a neutral flux limiter with value 1.0 with ion temperature decay length of $10 \mathrm{~cm}$, neutrals lost to the core, the minimum temperature in the neutral thermal flux limiter set to $10 \mathrm{eV}$ and the first flight model. The agreement of some of these 

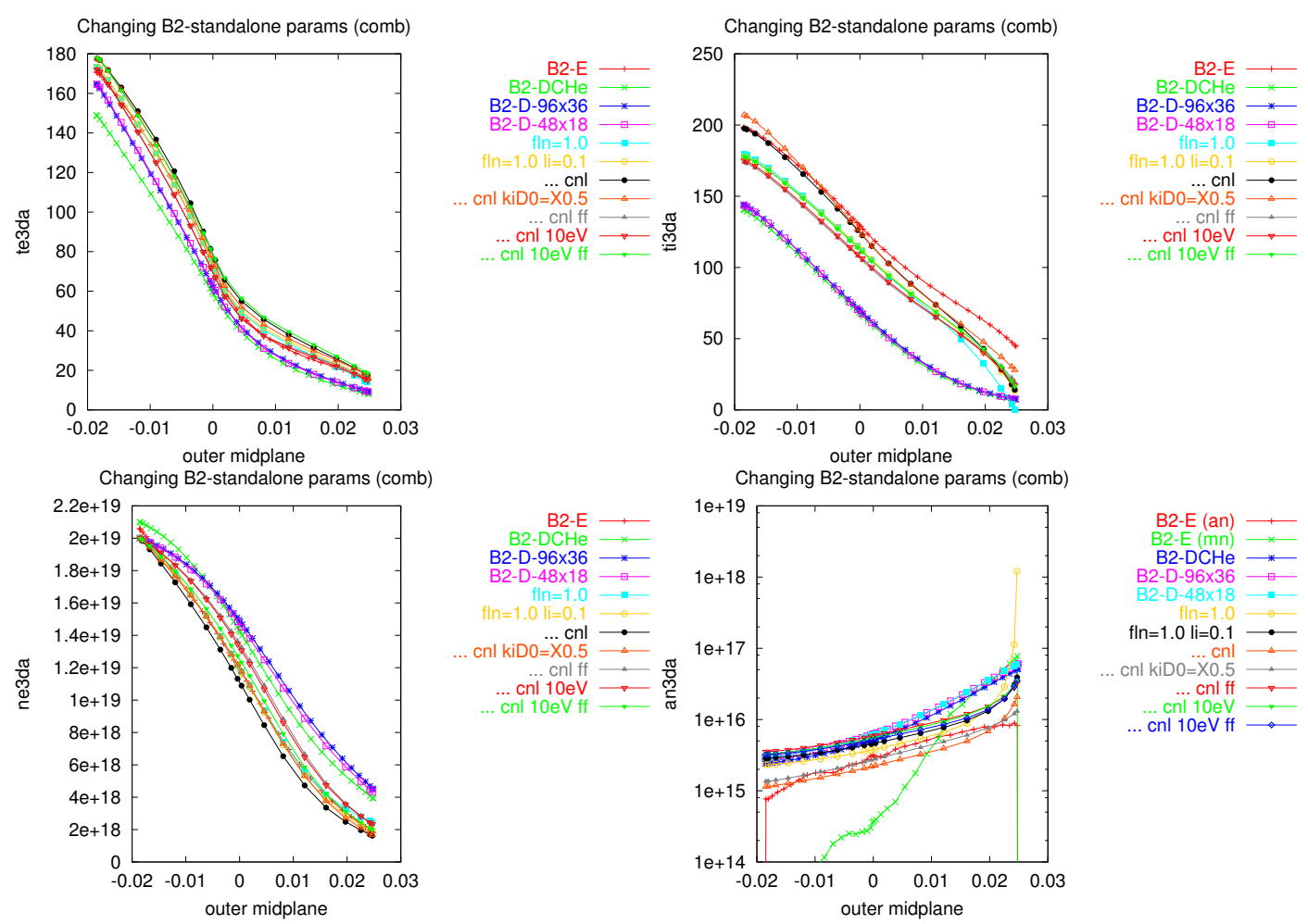

Fig. 10. Upstream electron temperature, ion temperature, electron density and neutral density. Data from the B2-Eirene run and the three B2 runs (with $\mathrm{D}+\mathrm{C}+\mathrm{He}$ on the same mesh, and then D on the same (96x36) mesh and a coarser mesh $(48 \times 18))$ are compared with seven B2-D-48x18 runs, with various combinations of previous variations. The now standard variation " $\mathrm{fln}=1.0$ " is combined with a larger ion temperature decay length, "fln=1.0 li=0.1", and then combined with enabling the loss of neutrals at the core (labeled "... cnl); this option is then also combined with a somewhat (factor 2) weaker neutral thermal diffusivity ( “... cnl kiD0=X0.5"), the first flight model ("... cnl ff"), a $10 \mathrm{eV}$ minimum temperature in the neutral flux limiter expression ("... cnl 10eV"), and then this last option combined with the first flight model ( $" . . . \mathrm{cnl} 10 \mathrm{eV}$ ff").

combinations is now quite good.

At a minimum, the recommendation for the fluid runs is: (1) use a core neutral loss boundary condition rather than a zero flux boundary condition, (2) use a neutral flux limiter with value 1.0, and (3) change the outer SOL ion temperature boundary condition to account for the neutral contribution to the "ion" thermal energy flux. The impact of the first-flight model is not so clear-cut. 

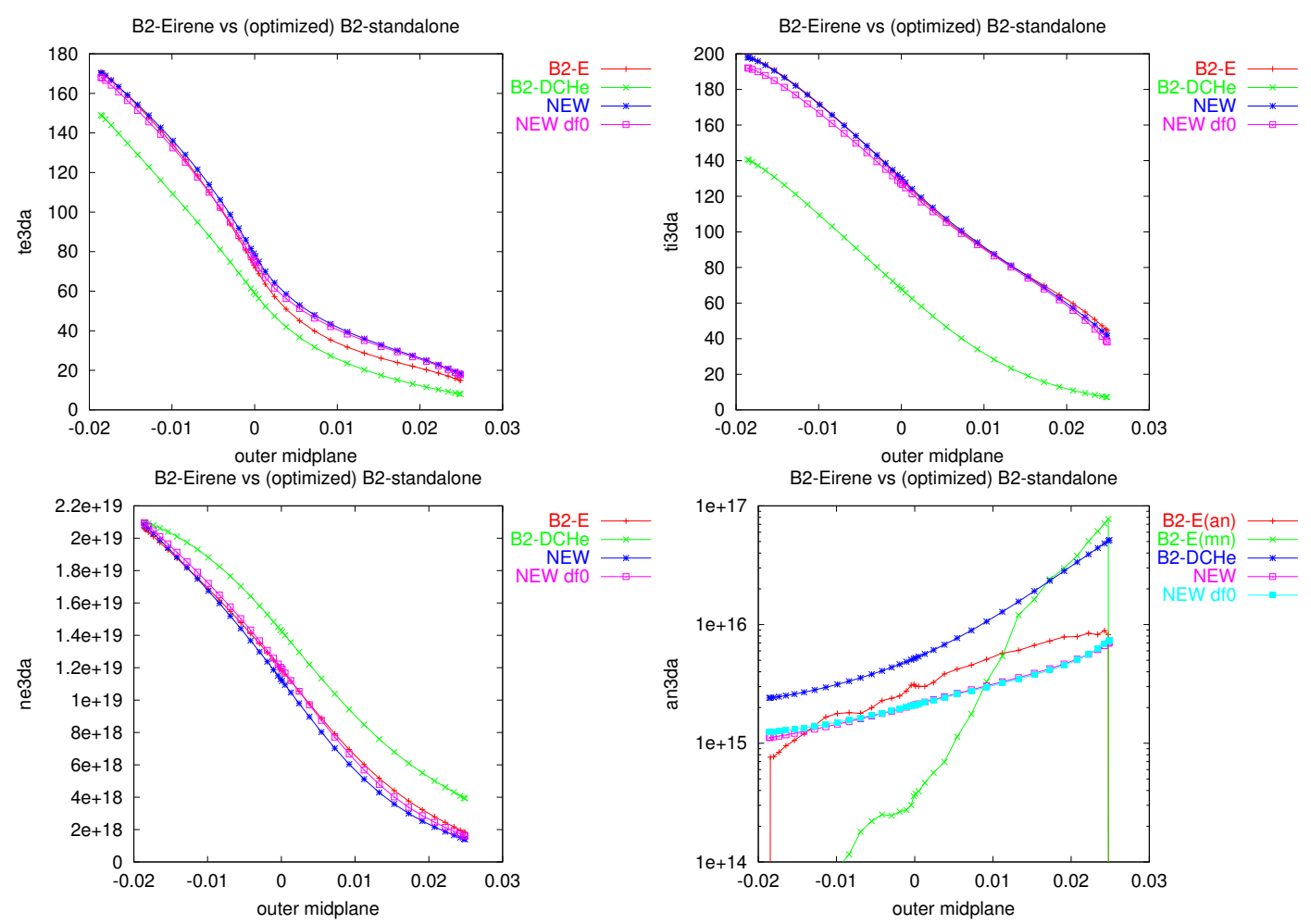

Fig. 11. Upstream electron temperature, ion temperature, electron density and neutral density. Data from the B2-Eirene run, the "original" B2-D $+\mathrm{C}+\mathrm{He}$ run and two "optimized" B2-D $+\mathrm{C}+$ He runs - the difference between the two "optimized" cases is that the second uses cross-sections based on atomic physics tables for determining the diffusivity in the fluid neutral model rather than constants. The agreement with the B2-Eirene runs for the two new optimized cases is significantly better than the starting point B2 run. A neutral flux limiter equal to 1.0, a core neutral loss term and a modified SOL ion temperature decay length $($ of $50 \mathrm{~cm}$ ) were used for the B2 runs.

\section{Re-doing the $\mathrm{D}+\mathrm{C}+\mathrm{He}$ fluid neutral calculations}

We now re-do the $\mathrm{B} 2 \mathrm{D}+\mathrm{C}+\mathrm{He}$ fluid neutral runs using the knowledge gained: (1) a core neutral loss term, (2) a neutral flux limiter of 1.0, (3) a relaxed SOL ion temperature decay length (of $50 \mathrm{~cm}$ in this case). The B2 standalone calculations are now a much better match to the B2-Eirene calculations, figure 11. A new variant is also presented, "NEW df0" which uses atomic physics rates from tables for calculating the neutral diffusivity, rather than constant cross-sections provided in the input file. 

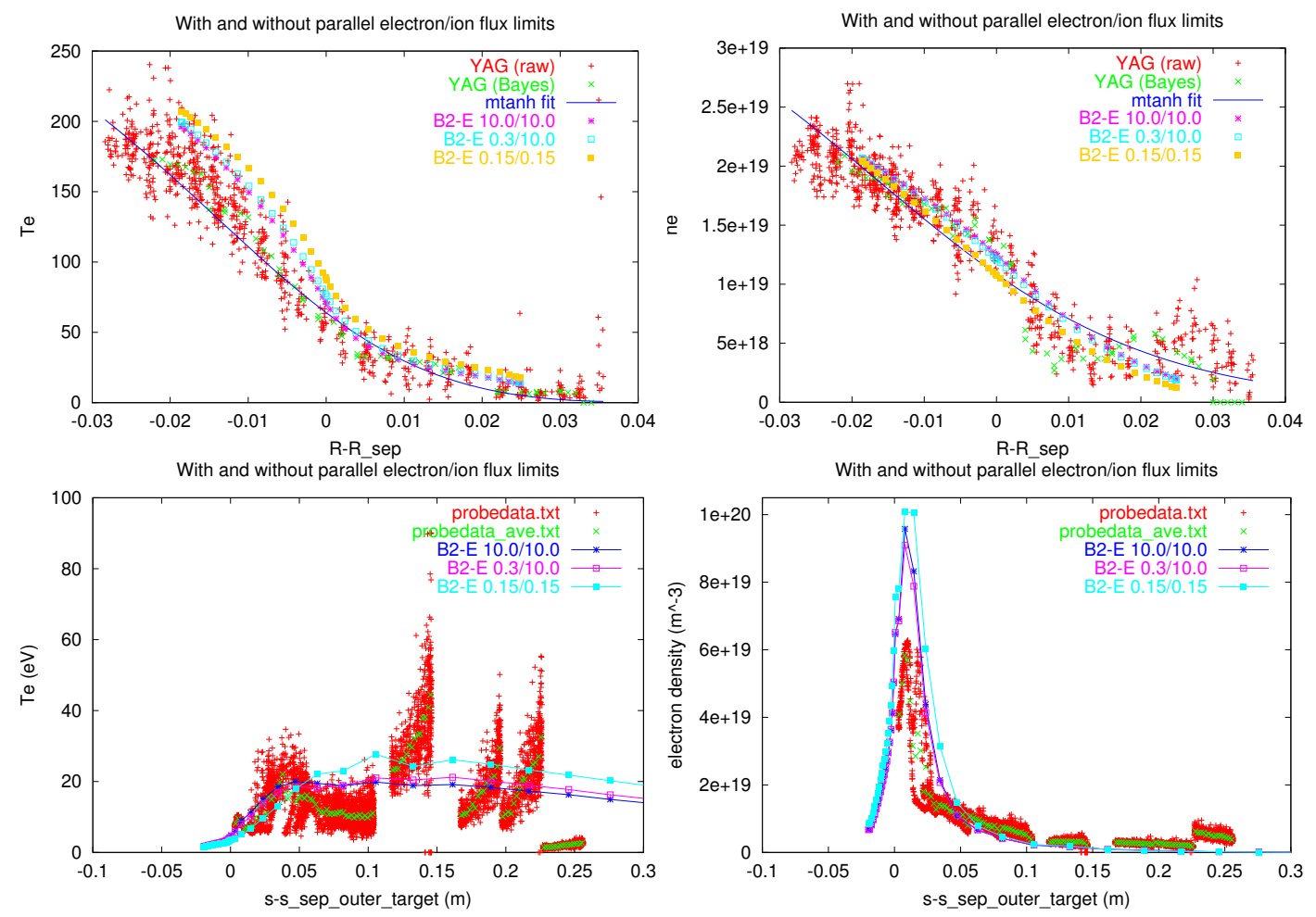

Fig. 12. Comparison of two B2-Eirene simulations with differing parallel heat flux limiters for electrons and ions with the experimental data. [The 0.3/10.0 case is identical to the B2-Eirene run discussed previously except that a slightly lower value of the perpendicular thermal diffusivities was used (1.2 instead of $\left.1.6 \mathrm{~m}^{2} \mathrm{~s}^{-1}\right)$.] Shown are the upstream electron temperatures and densities, and the downstream electron and downstream densities. It would be impossible to determine the "correct" parallel heat flux limiters on the basis of these measurements for this shot.

\section{$5 \quad$ Electron and ion parallel heat flux limits}

Another issue which affects the modelling is the use of parallel electron and ion heat flux limiters $[6,13-21]$. These are used in the codes to transition smoothly from a collisional regime where the parallel heat fluxes are given by SpitzerHärm to a kinetically limited term as the collisionality drops. In figure 12 the effects of changing the heat flux limits from the 0.3/10.0 (electron heat flux limited to $0.3 n_{e} T_{e} v_{\text {th,e }}$, ion effectively not limited) to $0.2 / 0.2$ (both electrons and ion parallel heat fluxes strongly flux limited) are shown to be small. It would be impossible to choose the "right" value of the heat flux limiters on the basis of these measurements for this shot.

For lower densities the heat flux limiters are expected to become more important, and this is borne out in figure 13 where the B2-Eirene simulations were repeated for a lower core deuterium density boundary condition. Under these circumstances, if it were possible to operate ASDEX Upgrade in L-mode 

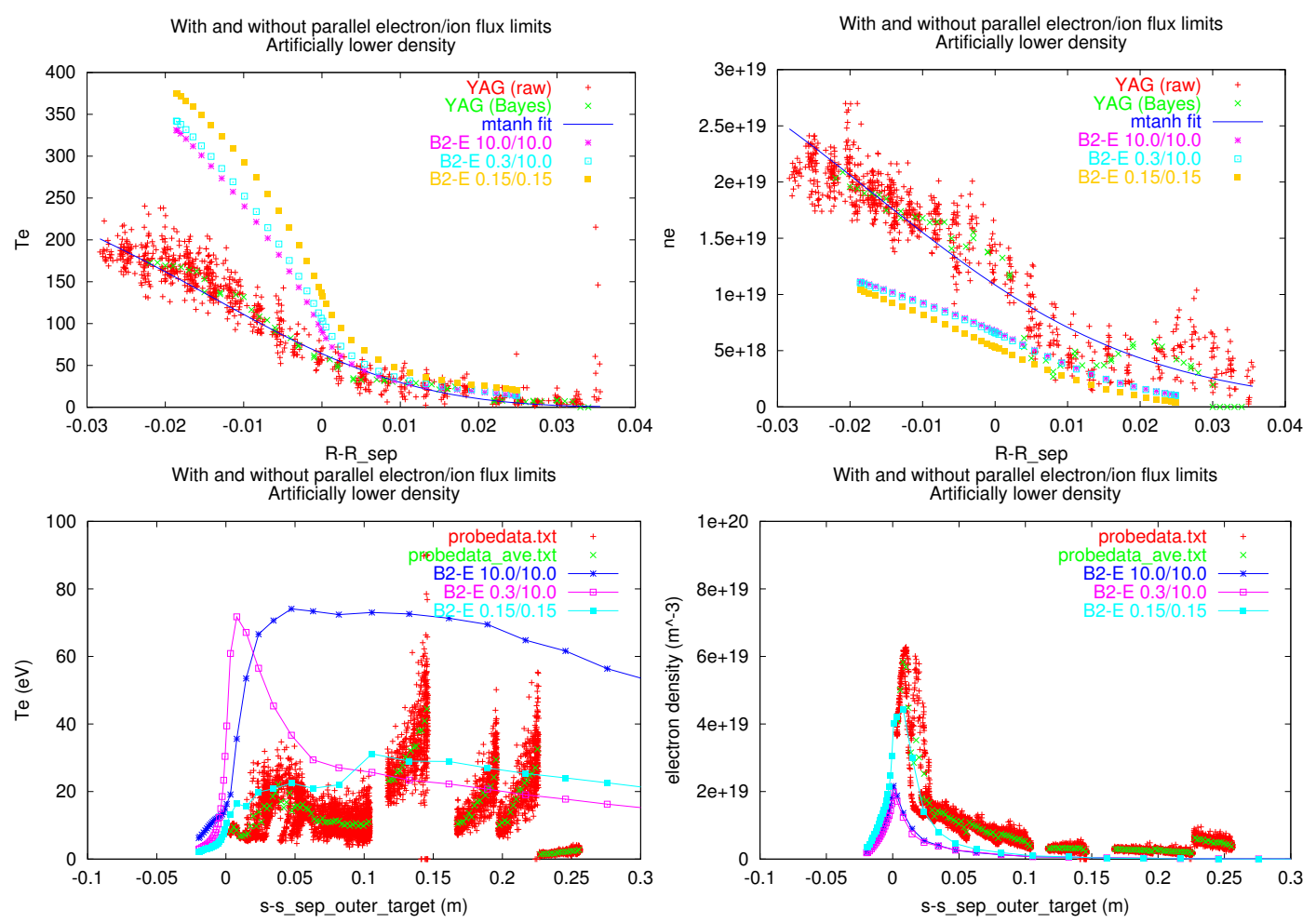

Fig. 13. Comparison of two B2-Eirene simulations with differing parallel heat flux limiters for electrons and ions with the experimental data, for a case where the densities in the B2-Eirene case were lowered (the deuterium density boundary condition was halved with respect to the "best match" shown in figure 12). Shown are the upstream electron temperatures and densities, and the downstream electron and downstream densities. The difference in the results for the simulations with differing values of the parallel heat flux limiters is now larger. Were the experiment able to operate in this regime on ASDEX Upgrade, it might be possible to distinguish the "correct" value of the flux limiters.

at these low densities, it might be possible to determine experimentally the "best" values for the parallel heat flux limiters (though at lower densities it might become more difficult to determine the power fraction into the electrons versus the ions).

The quantity most affected in the simulations by the change in the heat flux limiters is the ion temperature, figure 14, but the dearth of existing experimental data points to an opportunity!

\section{Ballooning transport}

From theoretical considerations it is expected that the transport level is higher on the outboard side (low field side, unfavourable curvature region) than on 

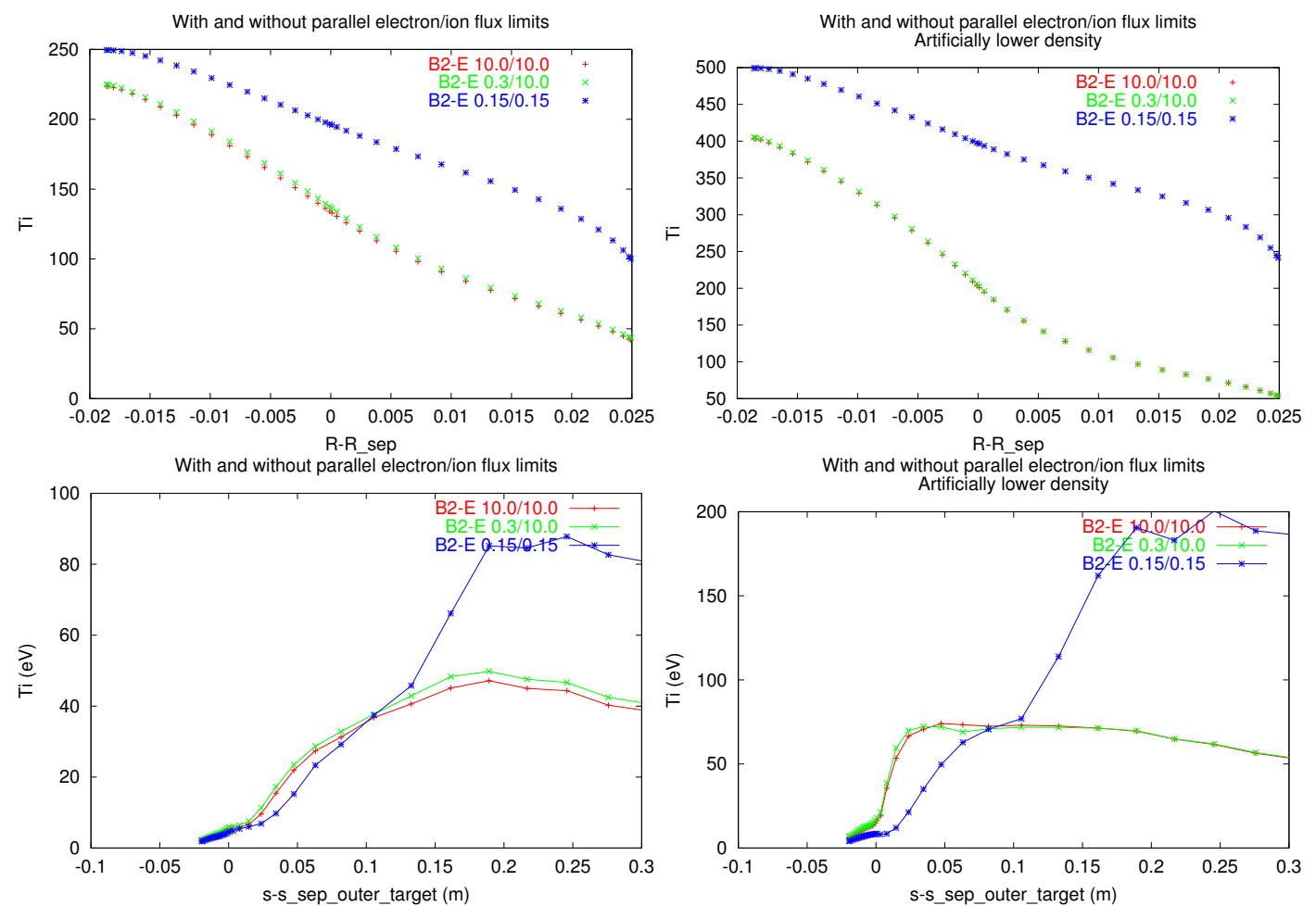

Fig. 14. Predicted ion temperatures upstream (top) and downstream (bottom) for the standard density case (left) and the (artificially) reduced density case (right). The ion temperatures show the strongest effect of the ion heat flux limiters - but there is no experimental data with which to compare (particularly at the target!).

the inboard side. The code runs discussed so far all have poloidally constant transport coefficients with the diffusion operator implemented in real space (which does give some ballooning because the flux surfaces are closer together on the outboard side). In figure 15 results are shown where the transport coefficients are made more ballooning. The resultant differences are, however, small, and on the basis of the current set of experimental measurements it would be impossible to determine the "appropriate" ballooning coefficient.

Even a comparison of the inner and outer target power fluxes, figure 16 would probably be inconclusive in determining the ballooning factor.

\section{Drifts}

The B2 code is also capable of including the drifts arising from $\vec{E} \times \vec{B}$ and $\nabla B$, and these terms (often not routinely included in code runs because of added numerical complications frequently forcing the use of a smaller time-step) are also important, figure 17. Here results for all four combinations of the plasma current and toroidal magnetic field are compared to a run without drifts, all for 

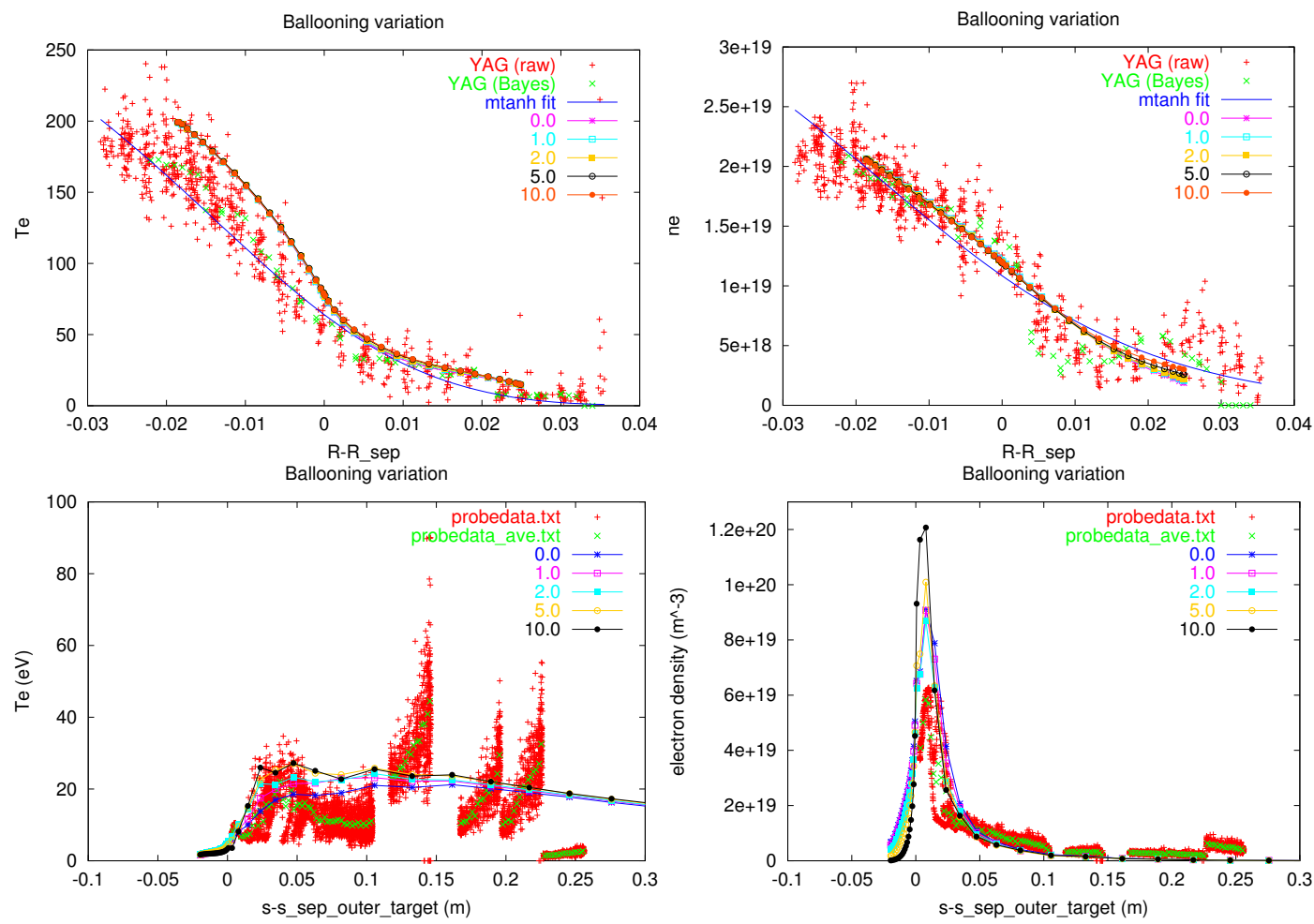

Fig. 15. Comparison of a case with the "normal" assumption of poloidally constant transport coefficients with cases where the transport is assumed to balloon on the outside. The transport is scaled locally by $\beta\left(B_{\text {ref }} / B\right)^{\alpha}$, where $B_{\text {ref }}$ is the magnetic field at a reference position, $B$ is the magnetic field at the local position, $\alpha$ is the ballooning parameter and is chosen to be 1.0, 2.0, 5.0 and 10.0, and $\beta$ is a normalization parameter chosen so the the "integrated" transport is held constant (by matching the core electron temperature profile). Shown are the upstream and downstream electron temperatures and densities. On the basis of these experimental measurements, it would not be possible to distinguish the "correct" value of the ballooning parameters, $\alpha$.
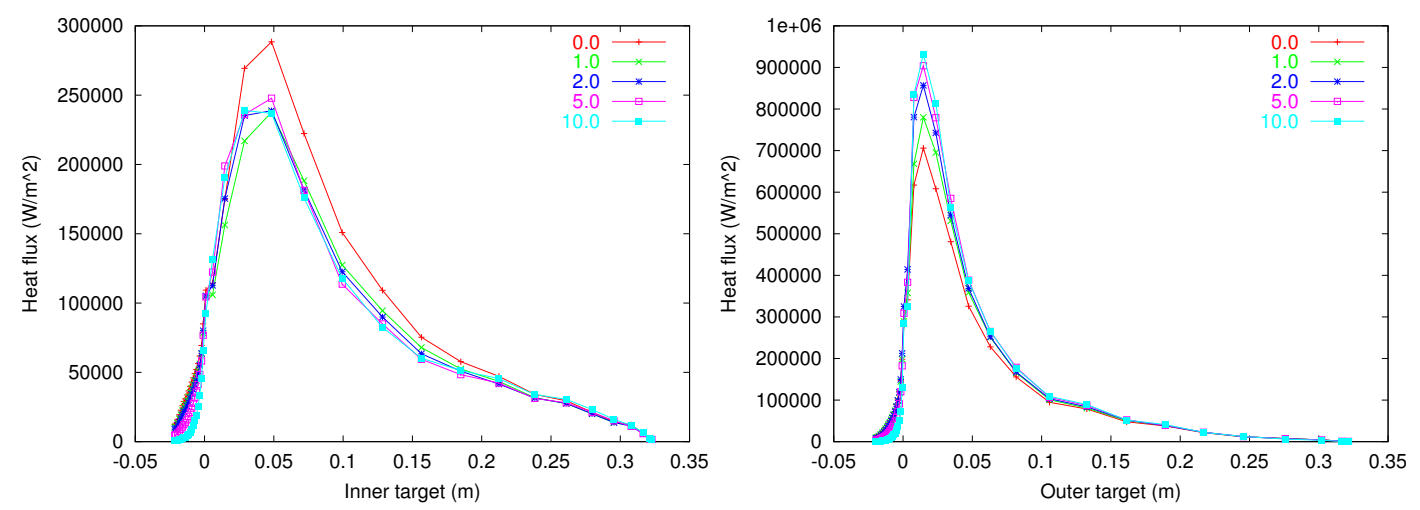

Fig. 16. Power fluxes to the inner and outer targets for the ballooning study of figure 15. 

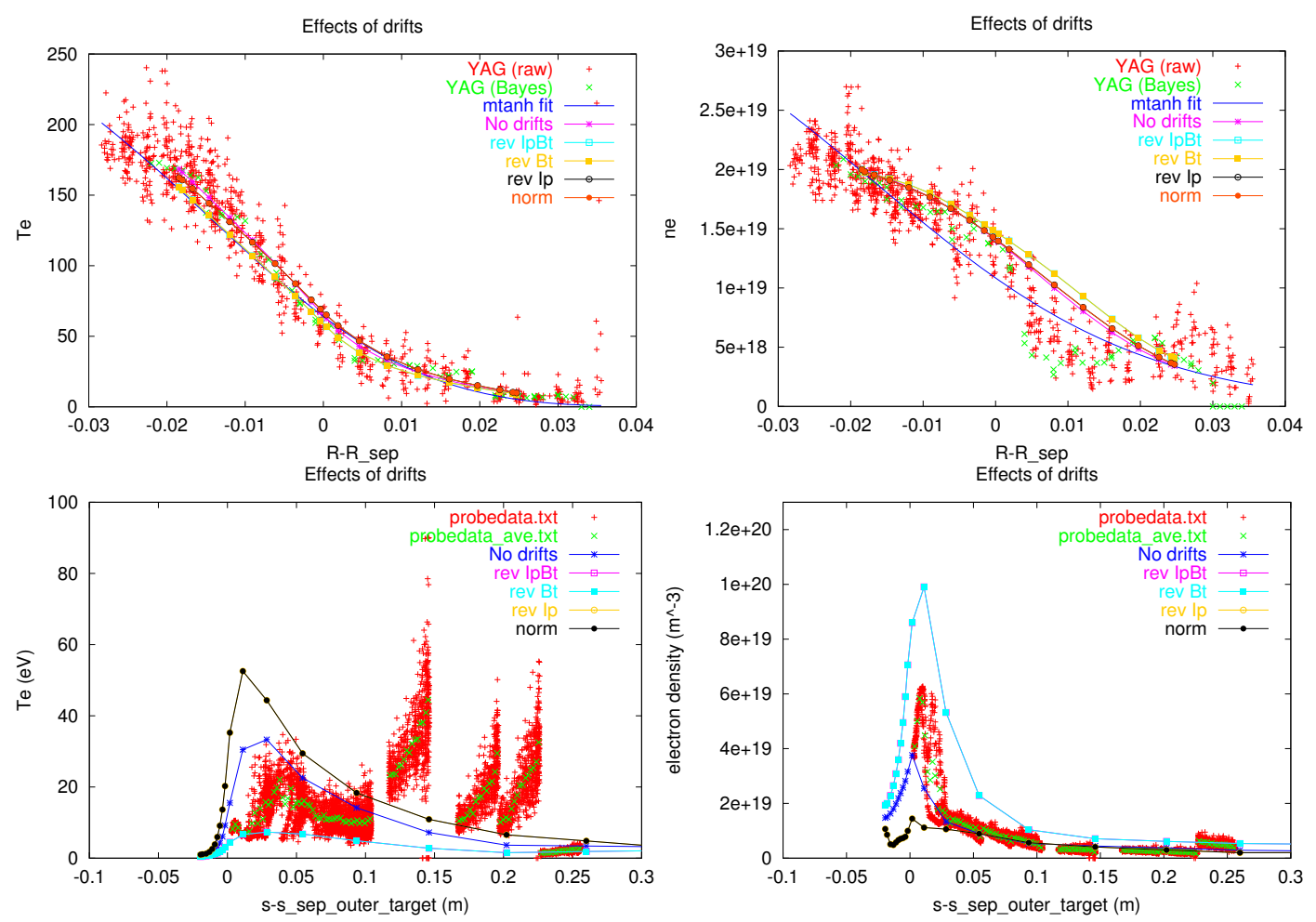

Fig. 17. Comparison of B2 standalone simulations and four simulations with drifts, corresponding to the four combinations of reversing the toroidal field and the plasma current.

pure deuterium plasmas on the reduced mesh. Only small differences are seen in the upstream profiles, but the downstream profiles are significantly affected. The final verdict on the match between the target experimental results and the code runs should wait for $\mathrm{D}+\mathrm{C}+\mathrm{He}$ runs with drifts using the coupled version of the code. (This is planned.)

\section{Conclusions and discussion}

We have shown that a fluid neutral model can reasonably accurately reproduce the upstream profiles of electron and ion temperatures, and electron and neutral densities, and a list of recommendations of how to achieve this in terms of the parameters of the fluid neutral model were presented. In addition the effects of parallel ion and electron parallel heat flux limiters, and transport ballooning to the low field side were small for the experimental conditions examined. The effect of including drift effects was shown to be significant for the target profiles.

The physics models in the codes have a number of "free" parameters. Some, 
such as those in the fluid neutral model, can be calibrated against more complete models - in the example against a kinetic neutrals model. Other "free" parameters, such as the electron and ion parallel heat flux limiters, could be calibrated by comparison with a full kinetic model of the edge plasma (were one to exist), or against the experiment. In the particular experimental shot used in this investigation, no strong influence was seen of these parallel flux limiters (or of the "ballooning" ansatz for the anomalous radial transport), and so no strong statement about them can be made. Or, looked at more positively, they did not affect the results and so were unimportant for the particular simulation of this shot.

Only very close interaction between experiment, modelling and theory (where modelling plays the mediating between experiment and theory) is able to improve the physics understanding of the edge plasma in tokamaks.

\section{Acknowledgments}

The authors would like to thank (1) Roberto Pugno, Lorne Horton, Arne Kallenbach and Ralf Dux for the experimental data; and (2) T. Rognlien and M. Umansky for technical discussions towards the implementation of neutral flux limits.

\section{References}

[1] R. Schneider, D. Reiter, H. P. Zehrfeld, B. Braams, M. Baelmans, et al., J. Nucl. Mater. 196-198 (1992) 810.

[2] D. Reiter, J. Nucl. Mater. 196-198 (1992) 80.

[3] D. Coster, X. Bonnin, K. Borrass, H.-S. Bosch, B. Braams, et al., in Proc. of the 18th IAEA Conference, Fusion Energy, Sorrento, Italy, October 2000, (CD-ROM), pages IAEA-CN-77/EXP5/32, Vienna, 2000, IAEA.

[4] D. Coster, X. Bonnin, B. Braams, H. Bürbaumer, E. Kaveeva, et al., in Proc. of the 19th IAEA Conference, Fusion Energy, Lyon, France, October 2002, (CD-ROM), pages IAEA-CN-94/TH/P2-13, Vienna, 2002, IAEA.

[5] B. J. Braams, Computational Studies in Tokamak Equilibrium and Transport, PhD thesis, Rijksuniversiteit, Utrecht, Nederland, 1986.

[6] B. J. Braams, Technical Report 68, Next European Torus, 1987.

[7] B. Braams et al., Contrib. Plasma Phys. 36 (1996) 276. 
[8] V. Rozhansky, S. Voskoboynikov, E. Kovaltsova, D. Coster, and R. Schneider, in Europhysics Conference Abstracts (CD-ROM), Proc. of the 26th EPS Conference on Controlled Fusion and Plasma Physics, Maastricht, 1999, edited by C. Bastian and C. Nieswand, volume 23J, pages 1749-1752, Geneva, 1999, EPS.

[9] R. Schneider, D. Coster, B. Braams, P. Xantopoulos, V. Rozhansky, et al., Contrib. Plasma Phys. 40 (2000) 328.

[10] V. Rozhansky, S. Voskoboynikov, E. Kovaltsova, D. Coster, and R. Schneider, Contrib. Plasma Phys. 40 (2000) 423.

[11] D. Reiter et al., J. Nucl. Mater. 220-222 (1995) 987.

[12] M. Umansky, T. Rognlien, M. Fenstermacher, M. Borchardt, A. Mutzke, et al., J. Nucl. Mater. 313-316 (2003) 559.

[13] R. C. Malone, R. L. McCrory, and R. L. Morse, Phys. Rev. Lett. 34 (1975) 71.

[14] A. R. Bell, R. G. Evans, and D. J. Nicholas, Phys. Rev. Lett. 46 (1981) 243.

[15] J. P. Matte and J. Virmont, Phys. Rev. Lett. 49 (1982) 1936.

[16] J. P. Matte, T. W. Johnston, J. Delettrez, and R. L. McCrory, Phys. Rev. Lett. 53 (1984) 1461.

[17] G. J. Rickard, A. R. Bell, and E. M. Epperlein, Phys. Rev. Lett. 62 (1989) 2687.

[18] Z. Abou-Assaleh, Kinetic Modelling of Edge Plasma in a Tokamak, PhD thesis, Université du Québec, 1991.

[19] D. Coster, Tokamak divertor modeling with fluid and kinetic codes, $\mathrm{PhD}$ thesis, Princeton University, 1993.

[20] M. Day, A Numerical Study of the Tokamak Edge Fluid Equations, PhD thesis, University of California, Los Angeles, 1995.

[21] W. Fundamenski, Tokamak Edge Plasma Modeling Using an Improved Onion-Skin Method, PhD thesis, University of Toronto, 1999. 